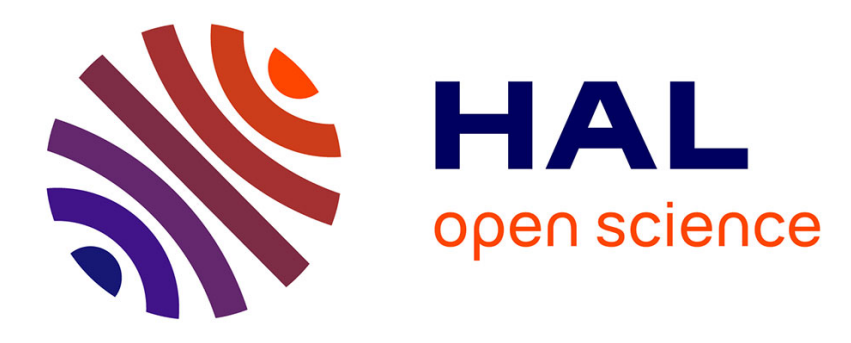

\title{
Navigating Dynamic Environments Using Trajectory Deformation
}

Vivien Delsart, Thierry Fraichard

\section{To cite this version:}

Vivien Delsart, Thierry Fraichard. Navigating Dynamic Environments Using Trajectory Deformation. IEEE-RSJ Int. Conf. on Intelligent Robots and Systems, Sep 2008, Nice, France. inria-00293505

\section{HAL Id: inria-00293505 https://hal.inria.fr/inria-00293505}

Submitted on 4 Jul 2008

HAL is a multi-disciplinary open access archive for the deposit and dissemination of scientific research documents, whether they are published or not. The documents may come from teaching and research institutions in France or abroad, or from public or private research centers.
L'archive ouverte pluridisciplinaire HAL, est destinée au dépôt et à la diffusion de documents scientifiques de niveau recherche, publiés ou non, émanant des établissements d'enseignement et de recherche français ou étrangers, des laboratoires publics ou privés. 


\title{
Navigating Dynamic Environments Using Trajectory Deformation
}

\author{
Vivien Delsart $^{\dagger}$ and Thierry Fraichard ${ }^{\dagger}$
}

\begin{abstract}
Path deformation is a technique that was introduced to generate robot motion wherein a path, that has been computed beforehand, is continuously deformed on-line in response to unforeseen obstacles. In an effort to improve path deformation, this paper presents a trajectory deformation scheme. The main idea is that by incorporating the time dimension and hence information on the obstacles' future behaviour, quite a number of situations where path deformation would fail can be handled. The trajectory represented as a space-time curve is subject to deformation forces both external (to avoid collision with the obstacles) and internal (to maintain trajectory feasibility and connectivity). The trajectory deformation scheme has been tested successfully on a planar robot with double integrator dynamics and a car-like vehicle.
\end{abstract}

Index Terms-Autonomous navigation; Motion deformation; Collision avoidance; Dynamic environments.

\section{INTRODUCTION}

Where to move next? is a key question for an autonomous robotic system. This fundamental issue has been largely addressed in the past forty years. Many motion determination strategies have been proposed (see [1] for a review). They can broadly be classified into deliberative versus reactive strategies: deliberative strategies aim at computing a complete motion all the way to the goal, whereas reactive strategies determine the motion to execute during the next few timesteps only. Deliberative strategies have to solve a motion planning problem. They require a model of the environment as complete as possible and their intrinsic complexity is such that it may preclude their application in dynamic environments. Reactive strategies on the other hand can operate on-line using local sensor information: they can be used in any kind of environment whether unknown, changing or dynamic, but convergence towards the goal is difficult to guarantee.

To bridge the gap between deliberative and reactive approaches, a complementary approach has been proposed based upon motion deformation. The principle is simple: a complete motion to the goal is computed first using a priori information. It is then passed on to the robotic system for execution. During the course of the execution, the still-tobe-executed part of the motion is continuously deformed in response to sensor information acquired on-line, thus accounting for the incompleteness and inaccuracies of the a priori world model. Deformation usually results from the application of constraints both external (imposed by the obstacles) and internal (to maintain motion feasibility and connectivity). Provided that the motion connectivity can be maintained, convergence towards the goal is achieved.

${ }^{\dagger}$ INRIA, CNRS-LIG \& Grenoble University, France.

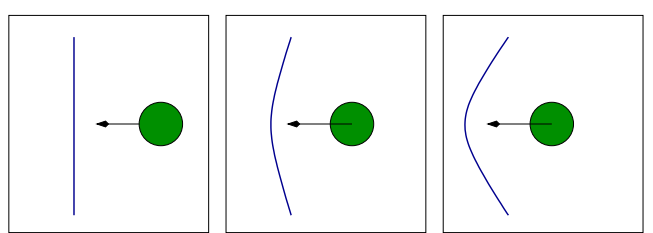

Fig. 1: Path deformation problem: in response to the approach of the moving disk, the path is increasingly deformed until it snaps (like an elastic band).

The different motion deformation techniques that have been proposed [2], [3], [4], [5], [6] all performs path deformation. In other words, what is deformed is a geometric curve, ie the sequence of positions that the robotic system is to take in order to reach its goal. The problem with path deformation techniques is that, by design, they cannot take into account the time dimension of a dynamic environment. For instance in a scenario such as the one depicted in Fig. 1, it would be more appropriate to leave the path as it is and adjust the velocity of the robotic system along the path so as to avoid collision with the moving obstacle (by slowing down or accelerating). To achieve this, it is necessary to depart from the path deformation paradigm and resort to trajectory deformation instead. A trajectory is essentially a geometric path parametrized by time. It tells us where the robotic system should be but also when and with what velocity. Unlike path deformation wherein spatial deformation only takes place, trajectory deformation features both spatial and temporal deformation meaning that the planned velocity of the robotic system can be altered thus permitting to handle gracefully situations such as the one depicted in Fig. 1.

The first trajectory deformation scheme has been proposed by one of the authors in [7]. It operates in two stages (collision avoidance and connectivity maintenance stages) and was geared towards manipulator arms. The contribution of this paper is a new trajectory deformation scheme, henceforth called Teddy (for Trajectory Deformer). It operates in one stage only and is designed to handle arbitrary robotic systems.

Teddy is designed to be one component of an otherwise complete autonomous navigation architecture. A motion planning module is required to provide Teddy with the nominal trajectory to be deformed. Teddy operates periodically with a given time period. At each cycle, Teddy outputs a deformed trajectory which is passed to a motion control module that determines the actual commands for the actuators of the robotic system. The paper focuses on Teddy 
only. It is organised as follows: Teddy is overviewed in $\S \mathrm{II}$ Its application to the case of a planar robot with double integrator dynamics is detailed in $\S \mathrm{III}$ whereas $\S \mathrm{IV}$ addresses the case of a car-like vehicle. Simulation results are then presented in $\S \mathrm{V}$

\section{OVERVIEW OF THE APPROACH}

\section{A. Notations and Definitions}

Let $\mathcal{A}$ denote a robotic system operating in a workspace $\mathbf{W}\left(\mathbb{R}^{2}\right.$ or $\left.\mathbb{R}^{3}\right)$. $q \in \mathbf{C}$ denote a configuration of $\mathcal{A}$. The dynamics of $\mathcal{A}$ is described by a differential equation of the form:

$$
\dot{s}=f(s, u)
$$

where $s \in \mathbf{S}$ is the state of $\mathcal{A}, \dot{s}$ its time derivative and $u \in \mathbf{U}$ a control. $\mathbf{C}, \mathbf{S}$ and $\mathbf{U}$ respectively denote the configuration space, the state space and the control space of $\mathcal{A}$. Let $\xi$ : $\left[0, t_{f}[\longrightarrow \mathbf{U}\right.$ denote a control input, ie a time-sequence of controls. Starting from an initial state $s_{0}$ (at time 0 ) and under the action of a control input $\xi$, the state of $\mathcal{A}$ at time $t$ is denoted by $\xi\left(s_{0}, t\right)$. A couple $\left(s_{0}, \xi\right)$ defines a trajectory for $\mathcal{A}$, ie a curve in $\mathbf{S} \times \mathbf{T}$ where $\mathbf{T}$ denotes the time dimension.

For the sake of trajectory deformation, a trajectory is discretized in a sequence of nodes. A node is a state-time, it is denoted by $n_{i}=\left(s_{i}, t_{i}\right)$. The discrete trajectory of $\mathcal{A}$ is $\Gamma_{0}=\left\{n_{0}, n_{1} \cdots n_{N}\right\}$ with $n_{0}$ (resp. $n_{N}$ ) the initial (resp. final) node of the trajectory.

\section{B. Trajectory Deformation Principle}

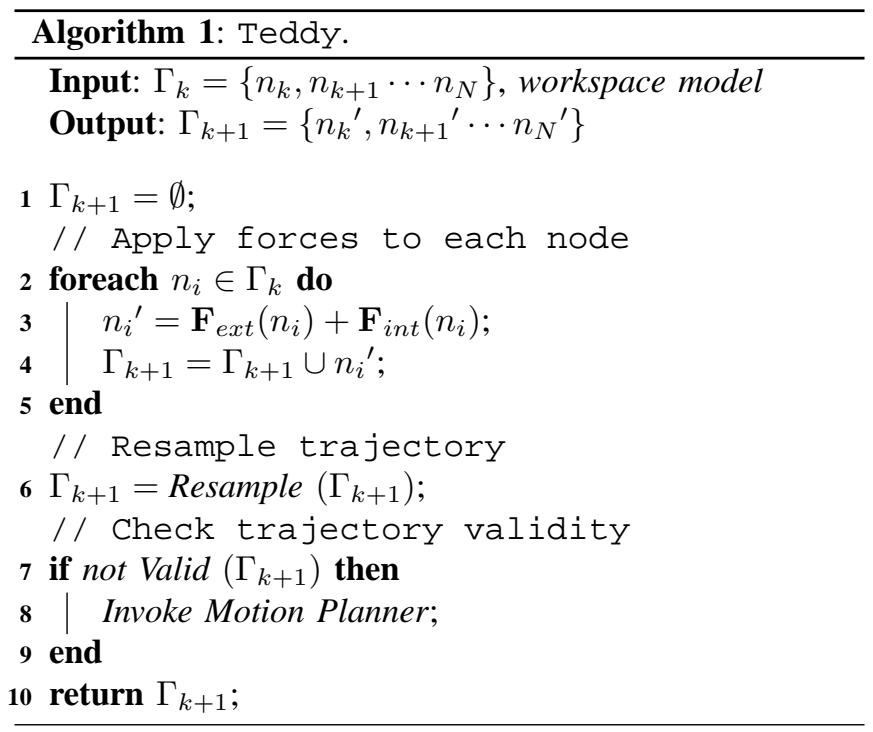

The main steps of Teddy are outlined in Algorithm 1. Teddy operates periodically with a time period of duration $T_{c}$. At time $t_{k}$, it takes as input the still-to-be-executed part of the trajectory $\Gamma_{k}=\left\{n_{k}, n_{k+1} \cdots n_{N}\right\}$ and an updated model of the workspace. The workspace model includes the position of the obstacles of $\mathbf{W}$ at time $t_{k}$ along with information about their future behaviour. Teddy then deforms $\Gamma_{k}$ in response to the updated position and future behaviour of the obstacles. At time $t_{k+1}=t_{k}+T_{c}$, Teddy outputs a deformed trajectory $\Gamma_{k+1}=\left\{n_{k}{ }^{\prime}, n_{k+1}{ }^{\prime} \cdots n_{N}{ }^{\prime}\right\}$ with $n_{i}{ }^{\prime}$ the updated node corresponding to $n_{i}$.

Like a particle placed in a force field, a node is displaced in response to the application of a force which is the combination of two kind of forces: external and internal. External forces (denoted $\mathbf{F}_{\text {ext }}$ ) are repulsive forces exerted by the obstacles of the environment, their purpose is to deform the trajectory in order to keep it collision-free. They are detailed in $\S$ II-C. Internal forces (denoted $\mathbf{F}_{\text {int }}$ ) on the other hand are aimed at maintaining the feasibility and the connectivity of the trajectory, ie to ensure that the deformed trajectory still satisfies the dynamics of $\mathcal{A}$. They are detailed in $\S$ III-D.

Now, for the sake of both collision-checking and connectivity evaluation, it is desirable to maintain a regular sampling level along the trajectory. Depending on the situation, nodes are removed or added accordingly. This point is detailed in $§ \mathrm{II}-\mathrm{E}$.

Finally, it is important to note that, like the path deformation scheme, the trajectory deformation scheme suffers from the following limitation: there is no guarantee that it will produce a collision-free and connected trajectory at each time step; both schemes are heuristic by nature. Failure to produce a valid trajectory typically happens when the topology of $\mathbf{S} \times \mathbf{T}$ changes (when a passage is blocked for instance, like when a door is closed). At each time step, the deformed trajectory is therefore checked for collision and connectivity. Should it become invalid, a global motion planner must be invoked to compute a new nominal trajectory. Strictly speaking, the motion planner is not part of Teddy, it is not discussed here.

\section{External Forces}

External forces are repulsive forces exerted by the obstacles of the environment for collision avoidance purposes. They are derived from a potential function $V_{\text {ext }}$. To explicitly take into account the future behaviour of the moving obstacles, $V_{\text {ext }}$ is defined in the space-time $\mathbf{W} \times \mathbf{T}$ (instead of $\mathbf{S} \times \mathbf{T}$ for efficiency reason). In a manner similar to [4], a set of points $p_{j}$ are selected on the body of $\mathcal{A}$. Each node $n_{i}$ of the trajectory $\Gamma_{k}$ yield a set of control points $c_{i}^{j}=\left(p^{j}, t_{i}\right)$ in $\mathbf{W} \times \mathbf{T}$. For a control point $c^{j}$ corresponding to the configuration $q$ and the state $s$ along the trajectory, $V_{\text {ext }}$ is defined as:

$$
V_{\text {ext }}\left(c^{j}\right)= \begin{cases}k_{e x t}\left(d_{0}-d_{w t}\left(c^{j}\right)\right)^{2} & \text { if } d_{w t}\left(c^{j}\right)<d_{0} \\ 0 & \text { otherwise }\end{cases}
$$

where $d_{w t}\left(c^{j}\right)$ is the distance from $c^{j}$ to the closest obstacle in $\mathbf{W} \times \mathbf{T} . d_{0}$ is the region of influence around the obstacles and $k_{e x t}$ is a repulsion gain. $d_{w t}$ is a distance function in $\mathbf{W} \times \mathbf{T}$. It is derived from the Euclidean distance by scaling the space versus the time dimension. In $\mathbb{R}^{2}$ for instance, the distance $d_{w t}$ between $\left(x_{0}, y_{0}, t_{0}\right)$ and $\left(x_{1}, y_{1}, t_{1}\right)$ is given by:

$$
\begin{aligned}
d_{w t}^{2}= & w_{s}^{2}\left(x_{1}-x_{0}\right)^{2}+w_{s}^{2}\left(y_{1}-y_{0}\right)^{2} \\
& +w_{t}^{2}\left(t_{1}-t_{0}\right)^{2}
\end{aligned}
$$


with $w_{s}$ (resp. $w_{t}$ ) the spatial (resp. temporal) weight. The force resulting from this potential function acting on $c^{j}$ is then defined as:

$$
\mathbf{F}_{e x t}^{w t}\left(c^{j}\right)=-\nabla V_{e x t}\left(c^{j}\right)=k_{e x t}\left(d_{0}-d_{w t}\left(c^{j}\right)\right) \frac{\mathbf{d}}{\|\mathbf{d}\|}
$$

where $\mathbf{d}$ is the vector between $c$ and the closest obstacle point. Now, $\mathbf{F}_{e x t}^{w t}$ has to be mapped into $\mathbf{S} \times \mathbf{T}$. The forces defined in $\mathbf{W} \times \mathbf{T}$ by each control point $c^{j}$ yield a force in $\mathbf{C} \times \mathbf{T}$ defined as follows:

$$
\mathbf{F}_{e x t}^{c t}(q, t)=\sum_{j=1}^{r} J_{c^{j}}^{T}(q, t) \mathbf{F}_{e x t}^{w t}\left(c^{j}\right)
$$

where $J_{c}^{T}(q, t)$ represents the Jacobian at point $c^{j}$ :

$$
J_{c^{j}}^{T}(q, t)=\left(\begin{array}{cccc}
\frac{\partial q^{1}}{\partial p_{1}^{j}} & \cdots & \frac{\partial q^{1}}{\partial p_{m}^{j}} & 0 \\
\vdots & \ddots & \vdots & \vdots \\
\frac{\partial q^{n}}{\partial p_{i}^{j}} & \cdots & \frac{\partial q^{n}}{\partial p_{m}^{j}} & 0 \\
0 & \cdots & 0 & 1
\end{array}\right)
$$

with $m$ the dimension of $\mathbf{W}, p_{l}^{j}$ the $l^{\text {th }}$ coordinate of $p^{j}$, $n$ the dimension of $\mathbf{C}$ and $q^{l}$ the $l^{t h}$ coordinate of $q$. The final mapping into $\mathbf{S} \times \mathbf{T}$ that yields $\mathbf{F}_{\text {ext }}(n)=\mathbf{F}_{\text {ext }}(s, t)$ is carried out by leaving the remaining parameters of $s$ unchanged.

\section{Internal Forces}

The external forces defined above push each node of the trajectory away from the obstacles if they are inside their influence region. Internal forces are introduced to ensure that the trajectory remains connected, ie that there exists a trajectory verifying the dynamics of $\mathcal{A}$ between two consecutive nodes of the trajectory. Trajectory connectivity is related to the concepts of forward and backward reachability. The set of states that are reachable from a given state $s_{0}$ are defined as (forward-reachability):

$$
\mathcal{R}\left(s_{0}\right)=\left\{s_{f} \in \mathbf{S} \mid \exists \xi, \exists t, s\left(s_{0}, \xi, t\right)=s_{f}\right\}
$$

Likewise, the set of states from which it is possible to reach a given state $s_{0}$ are defined as (backward-reachability):

$$
\mathcal{R}^{-1}\left(s_{0}\right)=\left\{s_{b} \in \mathbf{S} \mid \exists \xi, \exists t, s\left(s_{b}, \xi, t\right)=s_{0}\right\}
$$

Let $n_{-}, n$ and $n_{+}$denote three consecutive nodes of the trajectory $\Gamma_{k} . \Gamma_{k}$ is connected at $n$ iff $n \in \mathcal{R}\left(n_{-}\right)$and $n_{+} \in$ $\mathcal{R}(n)$. In other words, $n$ must belong to $\mathcal{R}\left(n_{-}\right) \cap \mathcal{R}^{-1}\left(n_{+}\right)$. Now, two cases arises depending on whether the intersection $\mathcal{R}\left(n_{-}\right) \cap \mathcal{R}^{-1}\left(n_{+}\right)$is empty or not (if this intersection is not empty, it means that $n_{-}$and $n_{+}$are connected together also). The next two sections detail how the internal forces are defined in both cases.
1) Case 1: $n_{-}$and $n_{+}$connected: In that case, the purpose of the internal force is to ensure that $n$ remains within $\mathcal{R}\left(n_{-}\right) \cap \mathcal{R}^{-1}\left(n_{+}\right)$. To that end, a virtual spring is defined between $n$ and a selected point $H$ belonging to $\mathcal{R}\left(n_{-}\right) \cap \mathcal{R}^{-1}\left(n_{+}\right)$. It yields a potential function $V_{\text {int }}$ defined in the space-time $\mathbf{S} \times \mathbf{T}$ as:

$$
V_{i n t}(n)=k_{i n t} d_{s t}(n)^{2}
$$

where $d_{s t}(n)$ is the distance between $n$ and $H$. It is defined in a manner similar to $d_{w t}$. $k_{\text {int }}$ is an attraction gain.

$$
\mathbf{F}_{\text {int }}(n)=-\nabla V_{\text {int }}(n)=k_{\text {int }} d_{s t}(n) \frac{\mathbf{d}}{\|\mathbf{d}\|}
$$

where $\mathbf{d}$ is the vector between $n$ and $H$.

2) Case 2: $n_{-}$and $n_{+}$disconnected: In that case, $\mathcal{R}\left(n_{-}\right) \cap \mathcal{R}^{-1}\left(n_{+}\right)=\emptyset$ and it is not possible to find a point $H$ belonging to $\mathcal{R}\left(n_{-}\right) \cap \mathcal{R}^{-1}\left(n_{+}\right)$. The solution proposed then is aimed at restoring the connectivity with $n_{-}$only. To that end, $H$ is simply selected within $\mathcal{R}\left(n_{-}\right)$and $\mathbf{F}_{\text {int }}$ is defined as in $\S$ II-D.1 above.

3) Selecting $H$ : Depending on whether $n_{-}$and $n_{+}$are connected together (ie whether $\mathcal{R}\left(n_{-}\right) \cap \mathcal{R}^{-1}\left(n_{+}\right)$is empty or not), $H$ should be selected within $\mathcal{R}\left(n_{-}\right) \cap \mathcal{R}^{-1}\left(n_{+}\right)$or $\mathcal{R}\left(n_{-}\right)$. In the former case, a natural choice for $H$ would be the centroid of $\mathcal{R}\left(n_{-}\right) \cap \mathcal{R}^{-1}\left(n_{+}\right)$. In the latter case, $H$ could for instance be defined as the point of $\mathcal{R}\left(n_{-}\right)$which is the closest to $n$.

Other choices are possible of course but the important thing to note is that, in theory, determining $H$ requires, in the worst case, the characterization of the three sets $\mathcal{R}\left(n_{-}\right), \mathcal{R}^{-1}\left(n_{+}\right)$and $\mathcal{R}\left(n_{-}\right) \cap \mathcal{R}^{-1}\left(n_{+}\right)$. Computing reachable sets for arbitrary robotic systems is a process whose complexity is dependent upon the dimensionality of the system considered and whether its dynamics is linear or not ( $c f$ [8], [9]). Since Teddy has a limited time $T_{c}$ only to deform the trajectory, it is therefore critical that Teddy be able to compute $\mathbf{F}_{\text {int }}(n)$ as efficiently as possible. To that end, it is important to exploit as much as possible the properties of the robotic system considered, and in some cases, to resort to various approximation or linearization schemes.

In the case where $n_{-}$and $n_{+}$are connected, another possibility is to compute a feasible trajectory from $n_{-}$to $n_{+}$ and to select, say its intermediate state, to define $H$. Once again, it is the particulars of the robotic systems at hand that determines how the internal forces are actually computed.

\section{E. Trajectory Resampling}

In the course of the deformation process, the nodes of the trajectory may either move away from their neighbours or, on the contrary, move very close to them (whether it be in the spatial or the temporal dimensions). For the sake of both collision-checking and connectivity evaluation, it is desirable to maintain a regular sampling level of the trajectory $\Gamma_{k}$. Depending on the situation, nodes are removed or added accordingly. 
Let $n_{-}, n$ and $n_{+}$denote three consecutive nodes of the trajectory $\Gamma_{k}$. A space-time distance similar to $d_{w t}$ is used to compute the distance between two nodes ( $c f(2))$. To begin with, if the distance between $n_{-}$and $n_{+}$is less than a given threshold, $n$ is removed from $\Gamma_{k}$. Then, the distance between $n_{-}$and $n$ is computed. If is is greater than a given threshold then a new intermediate node $n_{i}$ is added to $\Gamma_{k} . n_{i}$ can be defined as the centroid of $\mathcal{R}\left(n_{-}\right) \cap \mathcal{R}^{-1}(n)$. This nodeadding procedure is repeated recursively for both pair of nodes $\left(n_{-}, n_{i}\right)$ and $\left(\left(n_{i}, n\right)\right.$ (in case $n_{-}$and $n$ are really far from one another). The same node-adding procedure is repeated for the nodes $n$ and $n_{+}$.

\section{CASE Study 1: Double Integrator System}

\section{A. Model of the System}

To begin with, Teddy has been applied to the case of a 2D planar robot $\mathcal{A}$ with double integrator dynamics (point mass model). A state of $\mathcal{A}$ is characterized by $(p, v)$ that respectively denote the $2 \mathrm{D}$ position and velocity of $\mathcal{A}: p=$ $(x, y)$ and $v=\left(v^{x}, v^{x}\right)$. The dynamics of $\mathcal{A}$ is given by:

$$
\left(\begin{array}{c}
\dot{p} \\
\dot{v}
\end{array}\right)=\left(\begin{array}{l}
v \\
a
\end{array}\right)
$$

where $a$ denotes the acceleration control applied to $\mathcal{A}$ : $|a| \leq$ $a_{\max }$ and $|v| \leq v_{\max }$.

\section{B. Internal Forces Computation}

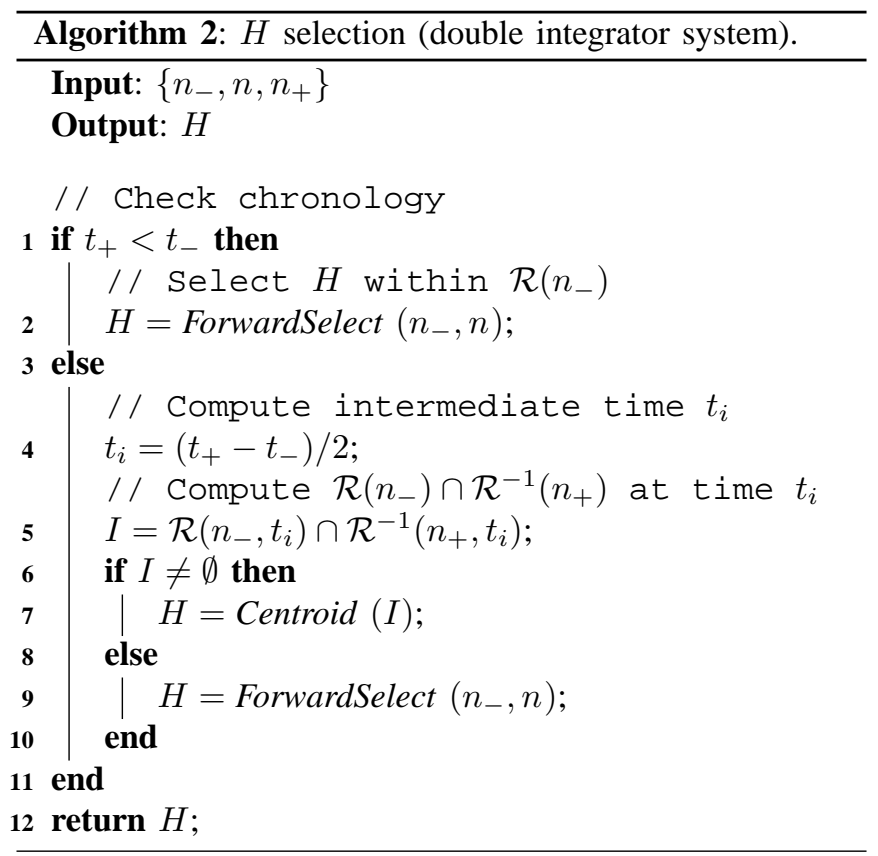

As mentioned earlier in $\S$ II-D.3, one key point in the adaptation of Teddy to a particular robotic systems lies in the determination of the point $H$ that is used to compute the internal force $\mathbf{F}_{\text {int }}$. It is important that $H$ can be computed efficiently. Algorithm 2 outlines the way $H$ is computed in this case. The main idea is to compute both $\mathcal{R}\left(n_{-}\right)$ and $\mathcal{R}^{-1}\left(n_{+}\right)$for a particular time slice only, namely the intermediate time slice $t=\left(t_{+}-t_{-}\right) / 2$. It is also taken advantage of the fact that, it is possible for the system (10) to compute the sets $\mathcal{R}\left(n_{-}\right), \mathcal{R}^{-1}\left(n_{+}\right)$and $\mathcal{R}\left(n_{-}\right) \cap \mathcal{R}^{-1}\left(n_{+}\right)$ for each spatial dimension independently.

Let us first consider how to compute $\mathcal{R}\left(n_{-}\right)$for the time slice $t$ (henceforth denoted $\mathcal{R}\left(n_{-}, t\right)$ ), and for the $x$ dimension only, $i e$ in the case where $n_{-}=\left(x_{-}, v_{-}^{x}, t_{-}\right)$. The $y$-dimension is dealt with similarly and so is the computation of $\mathcal{R}^{-1}\left(n_{+}, t\right)$.

1) Computing $\mathcal{R}\left(n_{-}, t\right)$ : First, the extremal positions reachable at time $t$ from $n_{-}$are computed. It is easily achieved by integrating forward (10) while applying the extremal control $\pm a_{\max }$ (until $\pm v_{\max }^{x}$ is reached). Let $p_{\min }(t)$ and $p_{\max }(t)$ denote these extremal positions. Then, for a discrete set of positions $p_{i} \in\left[p_{\min }(t) ; p_{\max }(t)\right]$, the corresponding extremal velocities $v_{\min }\left(p_{i}, t\right)$ and $v_{\max }\left(p_{i}, t\right)$ are computed. Now, the convex hull of the corresponding set of position-velocity pairs yields a $2 \mathrm{D}$ polygonal approximation of $\mathcal{R}\left(n_{-}, t\right) . \mathcal{R}^{-1}\left(n_{+}, t\right)$ is computed in a similar manner.

2) Computing $\mathcal{R}\left(n_{-}, t\right) \cap \mathcal{R}^{-1}\left(n_{+}, t\right)$ : $\operatorname{Both} \mathcal{R}\left(n_{-}, t\right)$ and $\mathcal{R}^{-1}\left(n_{+}, t\right)$ are represented by $2 \mathrm{D}$ polygons of the positionvelocity space. A straightforward polygon intersection yields $\mathcal{R}\left(n_{-}, t\right) \cap \mathcal{R}^{-1}\left(n_{+}, t\right)$.

3) Selecting $H$ : If $\mathcal{R}\left(n_{-}, t\right) \cap \mathcal{R}^{-1}\left(n_{+}, t\right)$ is not empty then its centroid is computed, it becomes $H$ (line 10 of Algorithm 2). Now, if $\mathcal{R}\left(n_{-}, t\right) \cap \mathcal{R}^{-1}\left(n_{+}, t\right)$ is empty, $H$ must be selected within $\mathcal{R}\left(n_{-}\right)$in order to try to maintain the connectivity between $n_{-}$and $n$. To that end, a discrete set of time instants $t_{j}>t_{-}$is defined and the corresponding reachable sets $\mathcal{R}\left(n_{-}, t_{j}\right)$ are computed as above. Their centroids $H_{j}$ are computed as well. Finally the point $H_{j}$ whose distance to $n$ is minimal becomes $H$ (lines 2 and 12 of Algorithm 2).

\section{Case Study 2: Car-Like System}

\section{A. Model of the System}

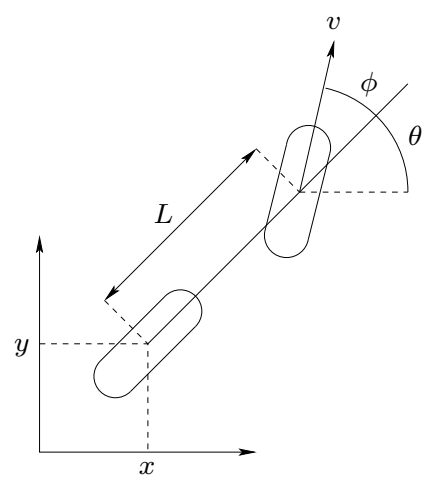

Fig. 2: The car-like vehicle $\mathcal{A}$ (bicycle model).

Teddy has then been applied to the case of a planar carlike vehicle $\mathcal{A}$. A state of $\mathcal{A}$ is characterized by $(x, y, \theta, \phi, v)$ where $(x, y)$ are the coordinates of the rear wheel, $\theta$ is the main orientation of $\mathcal{A}, \phi$ is the orientation of the front wheels (steering angle), and $v$ is the linear velocity of the front wheel (Fig. 2). A control of $\mathcal{A}$ is defined by the couple 
$u=(a, \zeta)$ where $a$ is the front wheel linear acceleration and $\zeta$ the steering velocity. The dynamics of $\mathcal{A}$ is given by:

$$
\left(\begin{array}{c}
\dot{x} \\
\dot{y} \\
\dot{\theta} \\
\dot{\phi} \\
\dot{v}
\end{array}\right)=\left(\begin{array}{c}
v \cos (\theta) \\
v \sin (\theta) \\
v \tan (\phi) / L \\
\zeta \\
a
\end{array}\right)
$$

where $L$ is the wheelbase of $\mathcal{A}$. It is assumed that $\mathcal{A}$ is moving forward only:

$$
v \in\left[0, v_{\max }\right],|\phi| \leq \phi_{\max },|a| \leq a_{\max } \text { and }|\zeta| \leq \zeta_{\max }
$$

\section{B. Internal Force Computation}

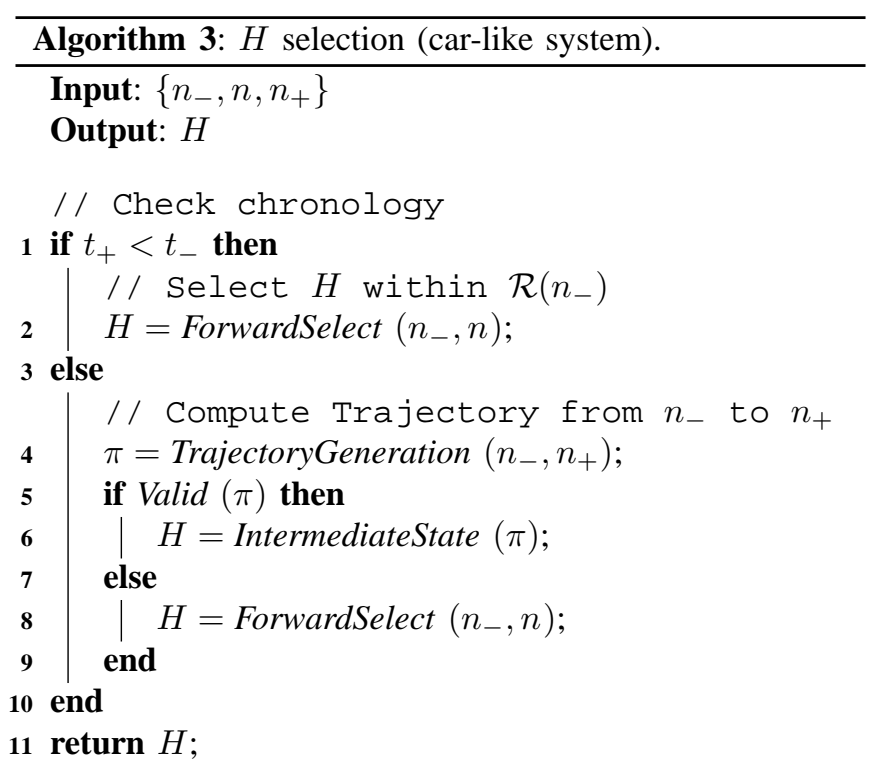

With its 5-dimensional state space and non-linear dynamics, the car-like system (11) has a complexity that prevents the efficient characterization of the reachable sets $\mathcal{R}\left(n_{-}\right), \mathcal{R}^{-1}\left(n_{+}\right)$and $\mathcal{R}\left(n_{-}\right) \cap \mathcal{R}^{-1}\left(n_{+}\right)$that are required in order to define $H$, the attraction state used in the definition of the internal force (9).

For efficiency reason, the following approach has been adopted instead in order to determine $H$ ( $c f$ Algorithm 3): a steering method is used to compute a feasible trajectory between $\left(n_{-}\right)$and $\left(n_{+}\right)$. If it succeeds, ie if $n_{-}$and $n_{+}$are connected, the intermediate state of the trajectory computed becomes $H$ and is used in the definition of $\mathbf{F}_{\text {int }}(n)$. Should the steering method fail to find a trajectory (when $n_{-}$ and $n_{+}$are not connected), an approximation of $\mathcal{R}\left(n_{-}\right)$is computed: the control space of $\mathcal{A}$ is randomly sampled and the corresponding states are computed using classical RungeKutta method [10]. The sample closest to $n$ becomes $H$.

The steering method used is derived from the trajectory generation method presented in [11]. It uses parametrized vehicle controls and nonlinear programming to search the control space for an optimum trajectory between two given states. It was modified in order to compute a trajectory to a goal state with a prescribed arrival time ( $c f[12])$. It achieves efficiency by using fast numerical optimization techniques and effective initial guesses for the control parameters.

\section{Simulation Results}

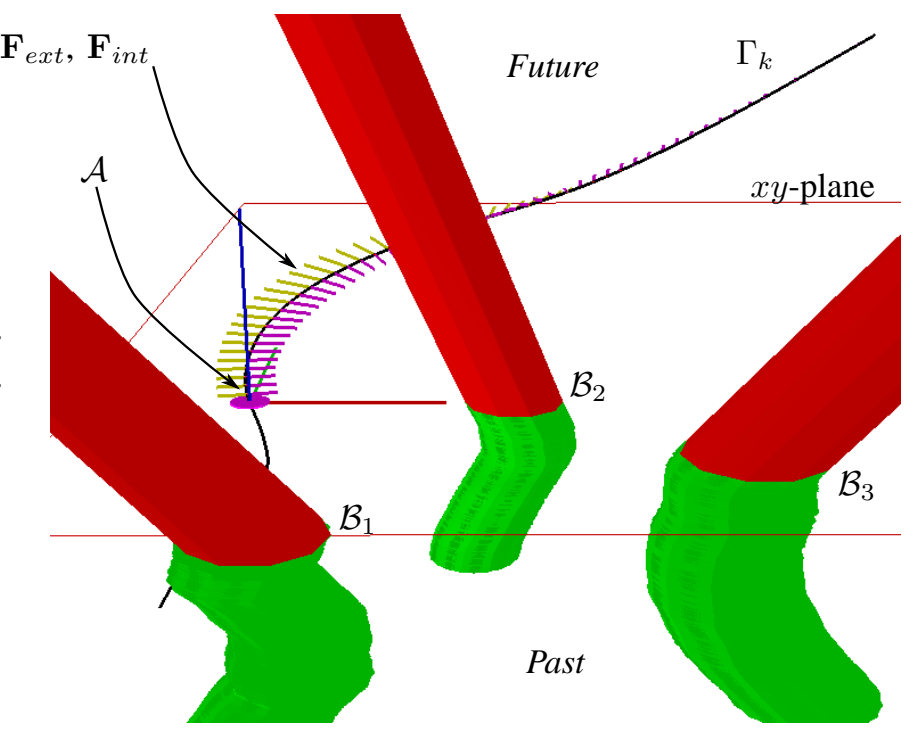

Fig. 3: Teddy's principle visualized in a scenario involving three moving disk obstacles $\mathcal{B}_{i}, i=1-3$. The time dimension is pointing upward. The past lies below the $x y$-plane (the present) and the future lies above. The obstacles are moving randomly but the model of the future assumes that they maintain a constant linear velocity. The internal and external forces acting upon the nodes of the trajectory $\Gamma_{k}$ are represented by vectors.

Teddy has been implemented in $\mathrm{C}++$ and tested on a desktop PC (Pentium 4@3GHz, 1GB RAM, Linux OS). Teddy has been evaluated in different scenarios featuring up to 40 circular obstacles moving randomly. At each time step, Teddy is provided with a new model of the environment that features the position and the geometry of the obstacles along with their current linear velocity (which is altered randomly at each time step). The model of the future is obtained by assuming that the obstacles maintain a constant linear velocity. This basic assumption is standard, it reflects the information that can be given by a tracking system able to determine the current position and speed of the moving obstacles. Teddy can of course handle more elaborate models of the future (using for instance long-term motion prediction models such as the ones developed by [13] among others). The important thing is that, at each time step, Teddy uses an updated workspace model and deforms the trajectory accordingly. Fig. 3 illustrates in a visual manner how Teddy operates.

The next two sections illustrates the workings of Teddy for the two robotics systems considered in different scenarios. The video which is attached to this paper presents similar results in a more lively fashion. 


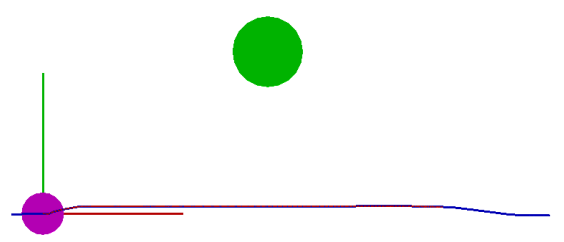

(a) space view, $t=0$

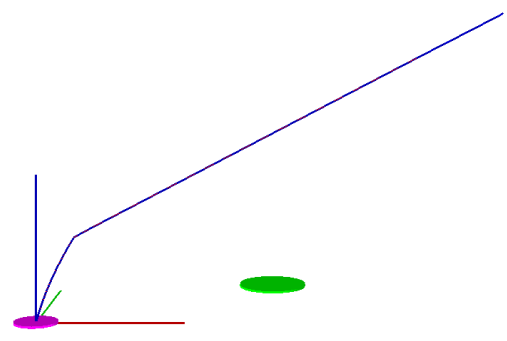

(d) time view, $t=0$

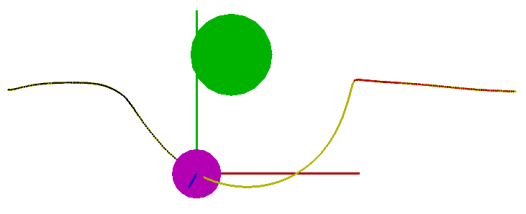

(b) space view, t $=10$

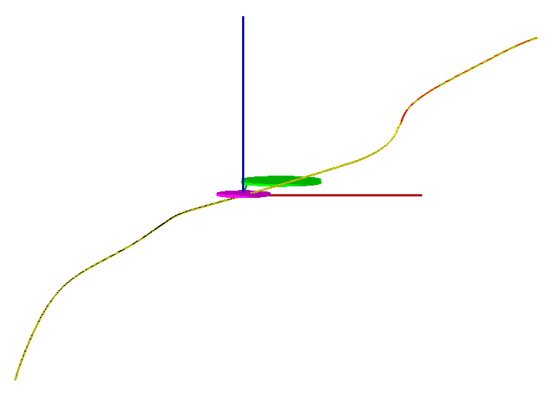

(e) time view, $t=10$

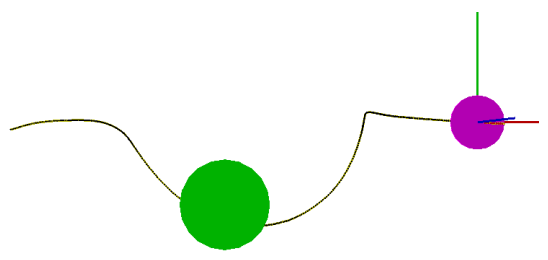

(c) space view, $t=20$

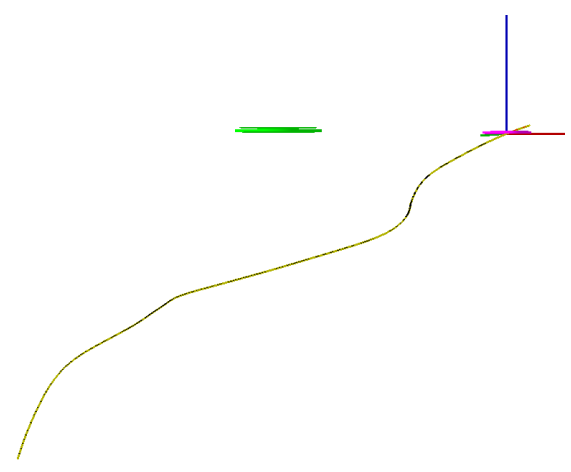

(f) time view, $\mathrm{t}=20$

Fig. 4: Double integrator system, "cutting" scenario (spatial deformation): $\mathcal{A}$ is moving from the left to the right, the obstacle is moving downwards. The top snapshots depict the path at different time instant $(x \times y$ view). The bottom snapshots depict the velocity profile at the same instants $(x \times t$ view).

\section{A. Double Integrator System}

To emphasize the interest of trajectory deformation $v s$ path deformation, a "cutting" scenario similar to the one depicted in Fig. 1 has been considered first. This scenario has been selected because it is problematic for classical path deformation schemes.

Teddy relies upon a number of parameters to operate properly: the repulsion gain $k_{e x t}$, the attraction gain $k_{i n t}$ and the distance functions $d_{w t}$ and $d_{s t}$. The two examples presented below have been selected to illustrate the importance of the distance function $d_{w t}$ on the performance of Teddy. Recall that $d_{w t}$ is used to determine the distance between a trajectory node and the closest obstacle in $\mathbf{W} \times \mathbf{T}(c f \oint \mathrm{II}-$ C). In both examples, the initial trajectory had a duration of 20 s and the discrete trajectory contained 320 nodes. Teddy would run at $28 \mathrm{~Hz}$.

For the same scenario, two very different deformation patterns can be obtained by properly selecting the weights $w_{s}$ and $w_{t}$ in (2). The first example is obtained by giving more weight to $w_{s}$ thereby allowing more important spatial deformations to take place (Fig. 4). In this case, $\mathcal{A}$ has time to pass before the obstacle crosses its path. The path component of the trajectory is deformed downwards for safety reasons whereas the velocity component is only slightly modified. The second example on the other hand is obtained by giving more weight to $w_{t}$ thereby allowing more important temporal deformations to take place (Fig. 5). In this case, $\mathcal{A}$ let the obstacle cross its path before proceeding. The path component of the trajectory is only slightly modified whereas the velocity component is largely deformed so as to allow $\mathcal{A}$ to slow down and stop in order to give way to the obstacle.

These two examples have shown the influence of the choice of the parameters in the final performance of Teddy. They have also illustrated the advantage of trajectory deformation versus path deformation. Afterwards, Teddy has been tested on different scenarios featuring both fixed and moving obstacles. The velocity of the moving obstacles change randomly at each time cycle. Such a scenario is depicted in Fig. 6. It features ten fixed and forty randomly moving obstacles.

\section{B. Car-Like System}

In the car-like system case, Teddy has also been tested on different scenarios featuring both fixed and moving obstacles. Fig. 7 depicts such a scenario wherein $\mathcal{A}$ is placed in an environment featuring ten fixed and twenty randomly moving obstacles. Fig. 7 illustrates the ability of Teddy to deform a trajectory while keeping its curvature and curvature derivative compatible with the dynamics of $\mathcal{A}$.

\section{Performances}

From a complexity point of view, the overall complexity of Teddy grows linearly with the number of nodes and 


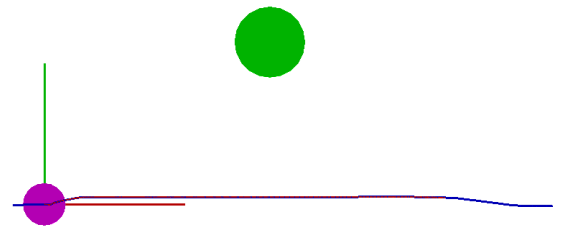

(a) space view, $t=0$

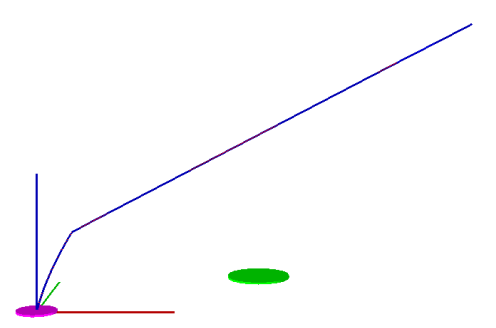

(d) time view, $\mathrm{t}=0$

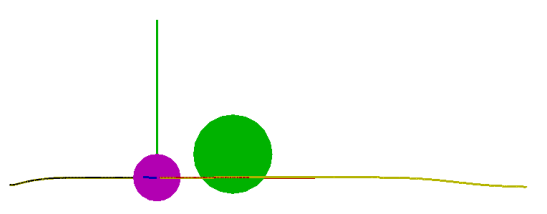

(b) space view, t $=10$

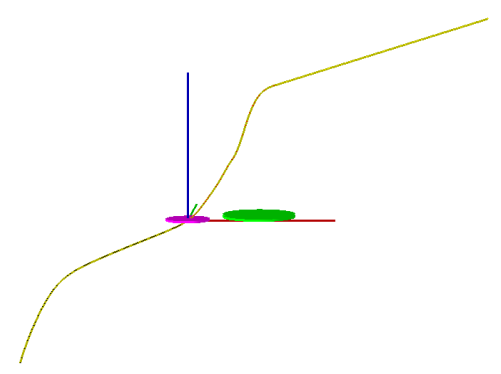

(e) time view, $\mathrm{t}=10$

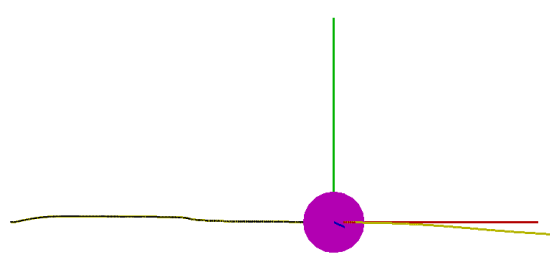

(c) space view, $t=35$

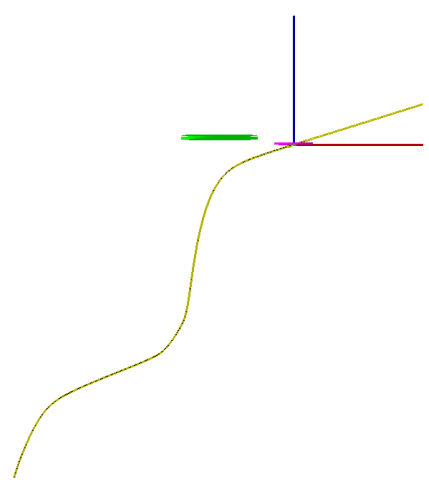

(f) time view, $\mathrm{t}=35$

Fig. 5: Double integrator system, "cutting" scenario (temporal deformation): $\mathcal{A}$ is moving from the left to the right, the obstacle is moving downwards. The top snapshots depict the path at different time instant $(x \times y$ view). The bottom snapshots depict the velocity profile at the same instants $(x \times t$ view).

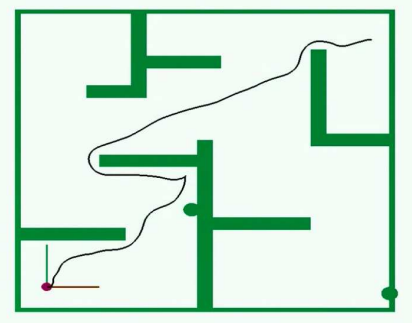

(a) $t=0$

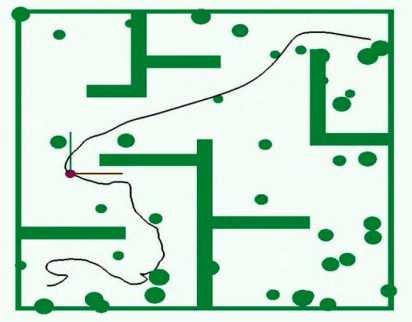

(d) $t=20$

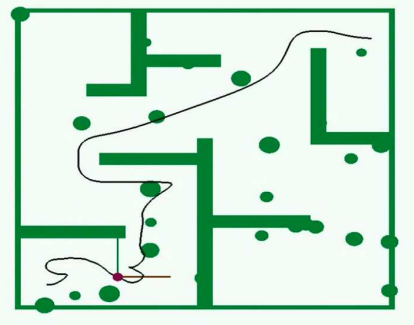

(b) $t=5$

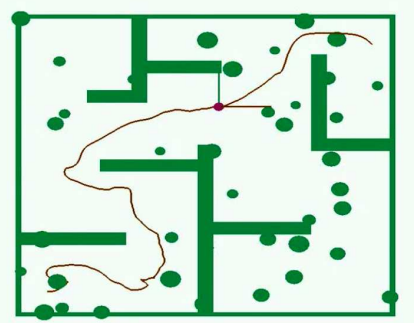

(e) $t=25$

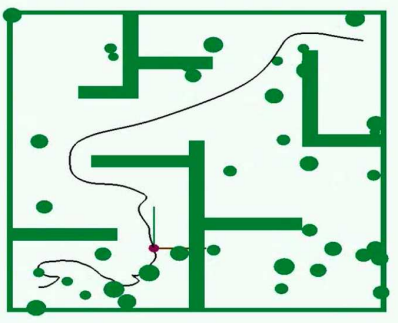

(c) $t=10$

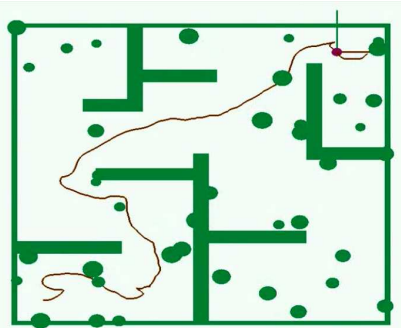

(f) $t=30$

Fig. 6: Double integrator system: the snapshots depict the path at different time instant $(x \times y$ view). 


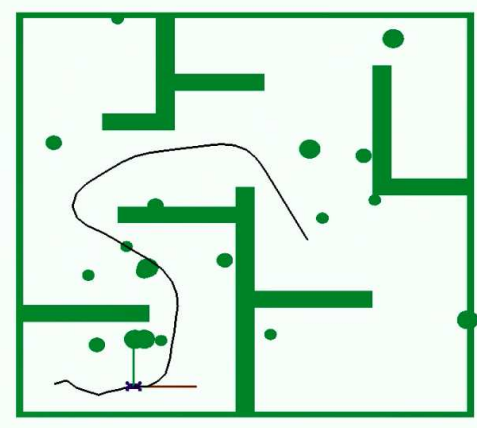

(a) $t=3$

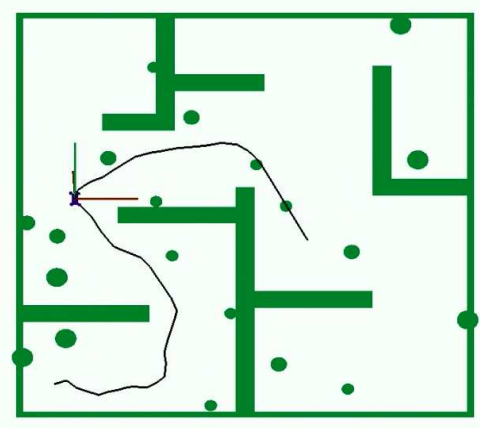

(b) $t=13$

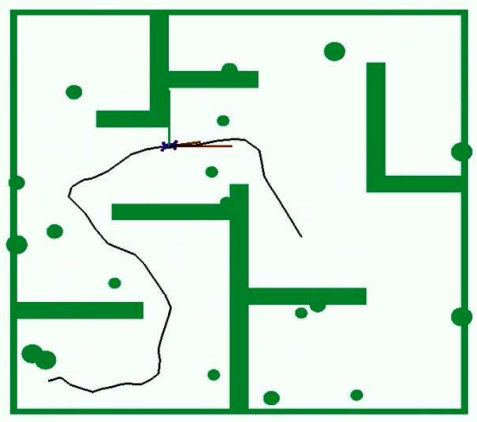

(c) $t=20$

Fig. 7: Car-like system: the snapshots depict the path at different time instant $(x \times y$ view).

\begin{tabular}{|c|c|c|c|c|c|}
\hline number of & \multicolumn{5}{|c|}{ number of nodes } \\
obstacles & 50 & 100 & 180 & 250 & 320 \\
\hline 1 & 6 & 11 & 20 & 27 & 35 \\
\hline 3 & 44 & 48 & 68 & 70 & 73 \\
\hline 10 & 49 & 88 & 135 & 199 & 229 \\
\hline
\end{tabular}

TABLE I: Running time (in ms) of one deformation cycle as a function of the number of nodes and obstacles.

the number of obstacles. Table I gives the running time for the double integrator system of one deformation cycle for different numbers of nodes and obstacles.

\section{CONCLUSION AND FUtURE WORKS}

The paper has presented Teddy, a trajectory deformation scheme. Given a nominal trajectory reaching a given goal, Teddy deforms it reactively in response to updated information about the environment's obstacles. Teddy can handle robotic systems with arbitrary dynamics. It has been applied to the case of a 2D double integrator system and a carlike system. Because, Teddy explicitly takes into account information on the future behaviour of the obstacles, it is able to handle situations that are problematic for classical path deformation schemes. In the future, it is planned to further optimize Teddy. Considering for instance that the knowledge about the future behaviour is less reliable in the distant future, it could be interesting to monotonically decrease the influence of the obstacles with respect to time. Last but not least, Teddy remains to be integrated within a global navigation architecture and tested on an actual robotic system. It is planned to do so on the architecture and the vehicle presented in [14]

\section{ACKNOWLEDGEMENTS}

This work has been supported by the French Ministry of Defence (DGA Doctoral Grant) and by the European Commission contracts "Cybercars-2 FP6-IST-2004-028062" and "Have-It FP7-IST-2007-212154".

\section{REFERENCES}

[1] S. M. Lavalle, Planning Algorithms. Cambridge University Press, 2006.

[2] S. Quinlan and O. Khatib, "Elastic bands: Connecting path planning and control," in Proc. of the IEEE Int. Conf. on Robotics and Automation, Atlanta, GA (US), May 1993.

[3] M. Khatib, H. Jaouni, R. Chatila, and J.-P. Laumond, "Dynamic path modification for car-like nonholonomic mobile robots," in Proc. of the IEEE Int. Conf. on Robotics and Automation, Albuquerque, NM (US), Apr. 1997.

[4] O. Brock and O. Khatib, "Elastic strips: a framework for motion generation in human environments," Int. Journal of Robotics Research, vol. 21, no. 12, Dec. 2002.

[5] F. Lamiraux, D. Bonnafous, and O. Lefebvre, "Reactive path deformation for nonholonomic mobile robots," IEEE Trans. on Robotics and Automation, vol. 20, no. 6, Dec. 2004.

[6] Y. Yang and B. Brock, "Elastic roadmaps: Globally task-consistent motion for autonomous mobile manipulation," in Proc. of the Int. Conf. Robotics: Science and Systems, Philadelphia PA (US), Aug. 2006.

[7] H. Kurniawati and T. Fraichard, "From path to trajectory deformation," in Proc. of the IEEE-RSJ Int. Conf. on Intelligent Robots and Systems, San Diego, CA (US), Oct. 2007.

[8] E. Asarin, T. Dang, G. Frehse, A. Girard, C. Le Guernic, and O. Maler, "Recent progress in continuous and hybrid reachability analysis," in Proc. of the IEEE Int. Conf. on Computer Aided Control Systems Design, Munich (DE), Oct. 2006.

[9] I. Mitchell, "Comparing forward and backward reachability as tools for safety analysis," in Hybrid Systems: Computation and Control, ser. Lecture Notes in Computer Science. Springer, 2007, no. 4416.

[10] C. Lubich, "On projected runge-kutta methods for differentialalgebraic equations," in BIT Numerical Mathematics, vol. 31, 1991.

[11] T. Howard and A. Kelly, "Optimal rough terrain trajectory generation for wheeled mobile robots," Int. Journal of Robotics Research, vol. 26, no. 2, Feb. 2007.

[12] V. Delsart and T. Fraichard, "Reactive trajectory deformation," INRIA," Research report, In Press.

[13] D. Vasquez, T. Fraichard, O. Aycard, and C. Laugier, "Intentional motion on-line learning and prediction," Machine Vision and Applications, Jan. 2008

[14] G. Chen, T. Fraichard, and L. Martinez-Gomez, "A real-time autonomous navigation architecture," in Proc. of the IFAC Symp. on Intelligent Autonomous Vehicles, Toulouse (FR), Sept. 2007. 